\title{
A Unified Approach to Phase Diagrams in Field Theory and Statistical Mechanics
}

\author{
Christian Borgs ${ }^{1}$ and John Z. Imbrie ${ }^{2 \star}$ \\ 1 Theoretische Physı, ETH-Hönggerberg, CH-8093 Zürich, Switzerland \\ 2 Lyman Laboratory of Physics, Harvard University, Cambridge, MA 02138, USA
}

\begin{abstract}
We construct the phase diagram of any system which admits a low-temperature polymer or cluster expansion. Such an expansion turns the system into a hard-core interacting contour model with small, but not necessarily positive, activities. The method uses some of Zahradnik's ideas [Z1], but applies equally well to systems with complex interactions. We give two applications. First, to low-temperature $P(\phi)_{2}$ models with complex couplings; and second, to a computation of asymptotics of partition functions in periodic volumes. If the index of a supersymmetric field theory is known, the second application would help determine the number of phases in infinite volume.
\end{abstract}

\section{Introduction}

In many systems in statistical mechanics and quantum field theory, the problem of competing ground states arises, even when the parameters of the model permit weak- or strong-coupling expansions. The standard example of such a system is the $N$-state Ising model at low temperatures (at or near first-order phase transitions.) In the absence of a symmetry between the states, one is faced with the problem of determining which states are thermodynamically stable. The successful theory of Pirogov and Sinai [PS] was developed to determine the stable phases of systems such as the $N$-state Ising model.

Subsequent authors developed the method for applications in more complicated systems. Quantum field theory models involving continuous space-time and continuous spins were handled by [I]. Statistical mechanics models with nontrivial structure within each phase were treated by [BKL, DZ], and others. In these studies, the need to use certain probability arguments from [PS] was a heavy burden. Especially in field theory, the combined requirements of decoupling and positive probability measures necessitated a very complicated procedure. Complex interactions were not accessible at all.

* Alfred P. Solan Research Fellow. Supported in part by the National Science Foundation under Grants PHY87-064220, DMS 88-58073, and PHY/DMS 86-45122 
Another important development of the theory was Zahradnik's method [Z1] which was conceptually much simpler than that of Pirogov and Sinai. The method was extended to systems with unbounded, continuous spins in [BW]. But the need for probability arguments still imposed unwanted restrictions on its applicability, and only very small complex parts could be permitted in the interactions (not uniform in the volume)--see [Z3]. Complex interactions were however permitted in the coarse-graining method of [GKK].

In the present work we extend Zahradnik's idea with a procedure that avoids the probability arguments, and hence applies equally well to complex interactions. A nice byproduct of the analysis is that signed contour-activities are allowed, and hence the generalizations involving interacting contours (quantum field theory, etc.) now fit under the same umbrella. Furthermore, complex couplings and nonpositive measures can now be permitted in the field theory models. In Sect. 4 we show how complex $P(\phi)_{2}$ models fit into the scheme, resulting in an approach which is at the same time more powerful and considerably simpler than that of [I].

Our starting point is a standard polymer system which arises any time one has a cluster expansion in a multiple phase model. We assume the usual estimates on such an expansion (small, exponentially decaying activities). The construction then produces a set of stable phases. If the model depends on some auxiliary parameters $\left\{\mu_{1}, \ldots, \mu_{N-1}\right\}$, then the usual phase diagram emerges, with hypersurfaces of codimension $k$ on which $k+1$ phases coexist.

Our procedure begins as in [Z1] by dropping all dangerous polymers/contours. These are ones for which the bounds needed to formulate a convergent cluster expansion fail (due to the formation of bubbles of the wrong phase). There are $N$ such truncated models (one for each possible boundary condition). One simply selects the phases for which the truncated free energy is minimal (i.e., it is less than or equal to all other truncated free energies). One can show that for these phases, the truncated model is the same as the original one, and hence its thermodynamic stability carries over to the original model.

To be specific, let $Z_{m}(V)$ denote the partition function in region $V$ with boundary condition $m \in\{1, \ldots, N\}$. Let $q \in\{1, \ldots, N\}$ be a state whose truncated free energy is minimal. Then we show that for any $V$ and any $m$,

$$
\left|\frac{Z_{m}(V)}{Z_{q}(V)}\right| \leqq e^{|\partial V|} .
$$

This condition of thermodynamic stability is well known as a sufficient condition for the convergence of a cluster expansion for correlation functions in the $q^{\text {th }}$ phase [I]. In the course of proving (1.1), we obtain a similar estimate for states with non-minimal free energy, but then only for volumes $V$ with diameter less than a certain critical length (see Theorem 3.1 below).

A nice feature of this method is the fact that one can immediately decide which are the stable phases. In contrast, the older methods determine the stable phases by a fixed point argument. The present method succeeds because the closer one gets to a coexistence of phases, the closer the truncated model comes to the true model. Hence it is sufficient to minimize the approximate free energies. To actually 
prove that the proposed set of stable phases is correct takes more work, and care must be taken in the complex case.

The paper is organized as follows. After defining the contour models in Sect. 2, we construct the stable phases and prove (1.1) in Sect. 3. Applications to $P(\phi)_{2}$ models and to periodic partition functions are given in the next two sections. The construction of the phase diagram is done in Sect. 6. In the complex case some difficulties arise because singularities can appear in the truncated free energies even though the initial model depends smoothly on all parameters. Nevertheless, we obtain differentiable phase coexistence hypersurfaces.

Supersymmetric models. One of our motivations for developing this procedure was a desire to understand the phase structure of supersymmetric field theory models - specifically the Wess-Zumino models studied by [JLW]. In the $N=1$ model the measure is signed, and so the method of [I] could not be used. We devote Sect. 5 to a study of the behavior of partition functions in periodic boxes. The asymptotics we obtain are especially interesting in the context of the index theorems proven by [JLW]. We assume a mean field expansion along the lines of [BG] has been constructed for these models. We then prove that the resulting polymer partition function on the torus $T$ has the following behavior for a large volume $|T|$ :

$$
\lim _{|T| \rightarrow \infty} Z^{P}(T) e^{f|T|}=n,
$$

where $n$ is the number of stable phases, and where $f$ is the free energy of the stable phases. We compare this with the index theorem proven in [JLW]:

$$
Z^{P}(T)=\operatorname{deg} V-1 \text {. }
$$

Here $V$ is the (polynomial) superpotential, and we consider for simplicity the $N=2$ case. Comparing (1.2) and (1.3), one sees immediately that $f=0$ (indicating no spontaneous supersymmetry breaking) and that $n=\operatorname{deg} V-1$ (which computes the number of stable phases).

We thus have a technique for demonstrating nonperturbatively the vanishing of the vacuum energy in each phase of the theory. Furthermore, coexistence of the $n$ phases is shown to be a consequence of supersymmetry (and an index theorem). This could be compared with using ordinary $\phi \rightarrow-\phi$ symmetry to prove coexistence of phases.

We consider also polymer models mimicking the signed $N=1$ measure-see Corollary 5.2. Then the right-hand side of (1.1) is replaced by a difference, with some stable phases contributing positively and some negatively. The sign corresponds to the effect we expect from the sign of the fermion mass. The index theorem is different in the $N=1$ case but some information on the number of stable phases can still be obtained.

We emphasize that these ideas will not come to fruition until the hard analysis on the Wess-Zumino models has been carried out. Also, we are necessarily limited to cases with deep, well separated minima. Still, (1.2) is interesting even in statistical mechanics, as it shows that each stable phase contributes equally to the periodic partition function (up to terms which vanish in the limit of large volume). 


\section{The Contour Models}

In this section we introduce the models for our phase structure analysis. These models are formulated slightly differently from the usual ones in order to nicely accommodate the case of Euclidean quantum field theory. The field theory models can be put into this form after development in a mean field cluster expansion, see Sect. 4.

We define the partition function in region $V$ with boundary condition $q \in Q=\{1,2, \ldots, N\}$. The index $q$ labels the "states" of the system, so that each of these $N$ possible boundary conditions has a chance of leading to a different thermodynamic pure state. The region $V$ is a finite union of unit cubes in $\mathbb{R}^{d}$, with $d \geqq 2$. The presence of boundary condition $q$ on $V$ will be indicated by $V^{q}$.

A configuration in $V^{q}$ will consist of a collection of contours (polymers, clusters) $Y_{\alpha}$, so we begin by defining these objects. A contour is a pair $(Y, q(\cdot))$, where $Y$ is a connected union of unit cubes (connectedness in the sense of sharing $(d-1)$ dimensional faces). The function $q(\cdot)$ is an assignment $q(F) \in Q$ (where $F$ indexes the faces in the boundary of $Y$ ) which, for any component $C$ of $Y^{c}=\mathbb{R}^{d} \backslash Y$, is constant on $\partial C$. The value of $q(\cdot)$ on the external boundary component of $Y$ plays a special role, and we sometimes emphasize this with a superscript $q$ on $Y$ and call $Y$ a $q$-contour. To simplify formulae, we let the symbols $Y$ or $Y^{m}$ denote the pair $(Y, q(\cdot))$ as well as the region $Y$. The finite components of $Y^{c}$ can be grouped according to boundary condition. We let $\operatorname{Int}_{m} Y$ denote the union of all finite components $C$ of $Y^{c}$ for which $q(\cdot)$ takes the value $m$ on $\partial C$, and write Int $Y=\bigcup_{m=1}^{N}$ Int $_{m} Y$. Finally, each contour $Y$ has a (possibly complex) translation-invariant activity $\rho(Y)$ satisfying the following bound for some large $\tau$ :

$$
|\rho(Y)| \leqq e^{-\tau|Y|}
$$

Here $|Y|$ denotes the volume of $Y$.

An allowed configuration of our system is a collection of nonoverlapping contours (no common cubes) with boundary condition compatibility. Compatibility is determined by the requirement that any connected component of $V \bigcup_{\alpha} Y_{\alpha}$ shall have constant boundary conditions. In addition, there is agreement on common boundaries, including $\partial V^{q}$. If the complement $V^{c}$ of $V$ is not connected, we do not allow contours whose interior intersects $V^{c}$. With a given collection of contours, the final element of the construction is an association of an energy density to regions in each of the phases of the model. A connected component of $V \backslash \bigcup_{\alpha} Y_{\alpha}$ that has boundary condition $m$ is considered to be part of $R_{m}$, the region "in the $m^{\text {th }}$ phase." Also, each $m$-contour is part of $R_{m}$. Thus we have partitioned $V$ as $\bigcup_{m} R_{m}$. We associate an energy density $e_{m}$ (which also may be complex) in the region $R_{m}$, and this yields the expression for the partition function:

$$
Z_{q}(V)=\sum_{\left\{Y_{\alpha}\right\}} \prod_{\alpha} \rho\left(Y_{\alpha}\right) \prod_{m=1}^{N} e^{-e_{m}\left|R_{m}\right|} .
$$


The connection between this partition function and the Peierls contour picture of spin systems is clear-we have just replaced sites with cubes and thickened contours to include neighboring cubes. The connection with field theory may be less clear, but we show how to put $P(\phi)_{2}$ models into this form in Sect. 4 . In brief, the cluster expansion converts the field theory partition into a polymer model like (2.2) with the multiphase structure reflected in the boundary conditions and the variable energy densities in (2.2). For field theory applications, it is important that the method be insensitive to the phase of $\rho(Y)$, since the cluster expansion inevitably produces nonpositive activities.

We introduce additional real parameters $\left\{\mu_{i}\right\}$ on which the activities $\rho$ and the energies $e_{q}$ may depend. There should be at least $N-1$ such parameters, and we need a degeneracy-breaking condition that the matrix

$$
\left(\frac{\partial}{\partial \mu_{i}} \operatorname{Re}\left(e_{q}-e_{N}\right)\right)_{q, i=1, \ldots, N-1}
$$

be nonsingular. We assume that $\rho$ and $e_{q}$ are $C^{1}$ functions of $\mu=\left(\mu_{1}, \ldots, \mu_{N-1}\right)$ with bounds

$$
\begin{aligned}
\left|\frac{\partial e_{q}}{\partial \mu_{i}}\right| & \leqq 1, \\
\left|\frac{\partial}{\partial \mu_{i}} \rho(Y)\right| & \leqq e^{-\tau|Y|} .
\end{aligned}
$$

The first condition sets a scale for the $\mu_{i}$, which play the role of generalized magnetic fields.

It is worth noting that the construction of the stable phases at a given set of parameter values $\left\{\mu_{i}\right\}$ does not require adjustments of the $\mu_{i}$ 's. Adjustments are needed only to locate those hypersurfaces at which various subsets of $Q$ are stable (see Sect. 6).

For many purposes we will need a second expression for $Z_{q}(V)$ which eliminates the compatibility of boundary conditions on contours. We resum (2.9) inside $\operatorname{Int}_{m}\left(Y^{q}\right)$, where $Y^{q}$ is an external contour of the set $\left\{Y_{\alpha}\right\}$ (i.e., it is not contained in Int $Y_{\alpha}$ for any $\alpha$ ). The resummation produces a factor $Z_{m}\left(\operatorname{Int}_{m} Y^{q}\right)$. (An external contour in $V^{q}$ must of course have boundary condition $q$.) This yields the expression

$$
Z_{q}(V)=\sum_{\left\{Y_{\alpha}^{\left.q_{3}\right\}_{\mathrm{x} 1}}\right.} \prod_{\alpha}\left[\rho\left(Y_{\alpha}^{q}\right) \prod_{m} Z_{m}\left(\operatorname{Int}_{m} Y_{\alpha}^{q}\right) e^{-e_{q}|V \backslash \mathrm{Int}|}\right],
$$

where the sum goes over sets $\left\{Y_{\alpha}^{q}\right\}_{\text {ext }}$ of mutually external contours, i.e., $Y_{\alpha} \cup \operatorname{Int} Y_{\alpha}$ and $Y_{\alpha^{\prime}} \cup$ Int $Y_{\alpha^{\prime}}$ do not overlap for $\alpha^{\prime} \neq \alpha$.

Also, we have denoted Int $=\bigcup_{m, \alpha} \operatorname{Int}_{m} Y_{\alpha}^{q}$. We devide each $Z_{m}$ by the corresponding $Z_{q}$ and multiply it back again in the form (2.6). Continuing this process, we obtain 


$$
\begin{aligned}
Z_{q}(V) & =e^{-e_{q}|V|} \sum_{\left\{Y_{\alpha}^{q}\right\}} \prod_{\alpha}\left[\rho\left(Y_{\alpha}^{q}\right) \prod_{m} \frac{Z_{m}\left(\operatorname{Int}_{m} Y_{\alpha}^{q}\right)}{Z_{q}\left(\operatorname{Int}_{m} Y_{\alpha}^{q}\right)}\right] \\
& \equiv e^{-e_{q}|V|} \sum_{\left\{Y_{\alpha}^{q}\right\}} \prod_{\alpha} K\left(Y_{\alpha}^{q}\right) .
\end{aligned}
$$

The only conditions on these $\left\{Y_{\alpha}^{q}\right\}$ are that the contours do not overlap, and that they all have outer boundary $q$. The expression (2.7) is useful for stable $q$ (defined in the next section) while (2.6) is better for unstable $q$ due to the possibility of zeros of $Z_{q}\left(\operatorname{Int}_{m} Y_{\alpha}^{q}\right)$.

\section{Determination of the Stable Phases}

In this section we will determine the stable phases of our model and show that for each stable phase $q$ (and for $\tau$ large enough)

$$
\left|K\left(Y^{q}\right)\right| \leqq e^{-(\tau-8 d)\left|Y^{q}\right|}
$$

which implies the convergence of cluster-expansions in the phase $q$. Since $K\left(Y^{q}\right)$ contains also partition functions with possibly unstable boundary conditions, we need a tool to deal with unstable partition functions. It is convenient and in fact much simpler than the original Pirogov-Sinai approach [PS] to use the notion of truncated contour models introduced by Zahradnik [Z1]. The idea is to truncate the sum (2.7) in such a way that it can be controlled by convergent cluster expansions. One then calculates the corresponding free energies $h_{q}$, and defines those boundary conditions $q$ to be stable, for which the real part of $h_{q}$ is minimal. In the last step one then shows that for stable boundary conditions the truncated partition function agrees with the untruncated one.

To motivate the following definitions we recall that $K\left(Y^{q}\right)$ is of the form

$$
K\left(Y^{q}\right)=\rho\left(Y^{q}\right) \prod_{m} f_{m}\left(Y^{q}\right)
$$

with

$$
f_{m}\left(Y^{q}\right)=\frac{Z_{m}\left(\operatorname{Int}_{m} Y^{q}\right)}{Z_{q}\left(\operatorname{Int}_{m} Y^{q}\right)},
$$

and that $|\rho(Y)| \leqq e^{-\tau|Y|}$. We call a contour $Y^{q}$ stable, if

$$
\left|f_{m}\left(Y^{q}\right)\right| \leqq \exp \left\{4\left|\partial \operatorname{Int}_{m} Y^{q}\right|\right\}
$$

for ail $m \in Q$. We define the truncated partition function $Z_{q}^{\prime}(V)$ as the partition function obtained from $Z_{q}(V)$ by leaving out the unstable contours:

$$
Z_{q}^{\prime}(V)=e^{-e_{q}|V|} \sum_{\left\{Y_{\alpha}^{q}\right\}}^{\prime} \prod_{\alpha} K\left(Y_{\alpha}^{q}\right) .
$$

Here the sum $\Sigma^{\prime}$ goes over sets $\left\{Y_{\alpha}^{q}\right\}$ of nonoverlapping, stable contours with outer boundary condition $q$. 
Definition 3.1. Let $h_{q}$ be the free energy of the truncated partition functions $Z_{q}^{\prime}$. Put

$$
a_{q}=\operatorname{Re} h_{q}-\min _{m \in Q} \operatorname{Re} h_{m}
$$

(we will sometimes write $a_{q}=a_{q}(\mu)$ to denote its dependence on the parameters $\mu_{i}$ ). If $a_{q}=0$, then the boundary condition $q$ is said to be stable.

From now on, we will use the letters $q, q^{\prime}$ for stable boundary conditions only, whereas $m, m^{\prime}$ may be arbitrary (stable or unstable) boundary conditions. Note that for an arbitrary contour $Y$

$$
\sum_{m}\left|\partial \operatorname{Int}_{m} Y\right| \leqq|\partial Y| \leqq 2 d|Y|,
$$

which shows that all stable contours obey the bound (3.1). Therefore, for $\tau$ large enough, the thermodynamic limit of $|V|^{-1} \log Z_{m}^{\prime}(V)$ can be controlled by a convergent cluster expansion. We conclude the existence of $h_{m}$, together with the bounds

$$
\begin{aligned}
\left|-\log Z_{m}^{\prime}(V)-h_{m}\right| V|| & \leqq O(\varepsilon|\partial V|), \\
\left|h_{m}-e_{m}\right| & \leqq O(\varepsilon),
\end{aligned}
$$

where we put $\varepsilon=e^{-\tau}$. Here, and elsewhere, we use $O(\varepsilon)$ for a bound const $\cdot \varepsilon$, with a constant that depends only on the number $N$ of possible $q$ and on the dimension $d$.

We now prove the following theorem, which implies that for $a_{q}=0$ all $q$-contours are stable. Thus $Z_{q}$ and $Z_{q}^{\prime}$ agree for stable boundary conditions.

Theorem 3.1. Assume that $|\rho(Y)| \leqq e^{-\tau|Y|}$ for all possible contours $Y$. Then, for $\tau>\tau_{0}$, where $\tau_{0}$ depends only on $d$ and $N$, the following statements hold:

i) If $a_{m} \operatorname{diam} V \leqq 1$ and $a_{q}=0$, then

$$
\left|\frac{Z_{q}(V)}{Z_{m}(V)}\right| \leqq e^{a_{m}|V|+|\partial V|},
$$

ii) If $a_{q}=0$, then $Z_{q}(V) \neq 0$ and

$$
\left|\frac{Z_{m}(V)}{Z_{q}(V)}\right| \leqq e^{|\partial V|},
$$

iii) If $a_{m} \operatorname{diam} V \leqq 1$, then $Z_{m}(V) \neq 0$ and

$$
\left|\frac{Z_{\tilde{m}}(V)}{Z_{m}(V)}\right| \leqq e^{3|\partial V|}
$$

for all $\tilde{m} \in Q=\{1, \ldots, N\}$.

Proof. To prove the theorem, we introduce the notion of small and large contours. We say that $a$ contour $Y^{m}$ is small if $a_{m} \operatorname{diam} Y^{m} \leqq 1$; it is large if $a_{m} \operatorname{diam} Y^{m}>1$. We also define the partition function $Z_{q}^{\text {small }}(V)$ which is obtained from $Z_{q}^{\prime}(V)$ by replacing the sum over stable contours in (3.5) by a sum over small contours. If we sum, instead, only over contours which are at the time small and stable, we denote the resulting partition function by $Z_{q}^{\prime \text { small }}$. 
We prove the theorem by induction on the diameter of $V$. So we assume that $\operatorname{diam} V=k$ and that i)-iii) have already been proven for all volumes $W$ with $\operatorname{diam} W<k$.

Proof of i) for $\operatorname{diam} V=k$. For any contour $Y$ in $V$, and any $\tilde{m} \in\{1, \ldots, m\}$, we have $\operatorname{diam~Int~}_{\tilde{m}} Y \leqq k-1$. We therefore can use the inductive assumptions ii) and iii) that all $q$-contours and all $m$-contours in $V$ are stable. Therefore

$$
\frac{Z_{q}(V)}{Z_{m}(V)}=\frac{Z_{q}^{\prime}(V)}{Z_{m}^{\prime}(V)}
$$

Using the convergence of the cluster expansion for $\log Z_{\tilde{m}}^{\prime}$ one immediately gets i). Proof of ii) for diam $V=k$. By the inductive assumption all $q$-contours in $V$ are stable. Therefore $Z_{q}(V)$ can be controlled by a convergent expansion (in particular $\left.Z_{q}(V) \neq 0\right)$. To control $Z_{m}(V)$ we rewrite it using the relation (2.6). Write a set $\left\{Y_{\alpha}^{m}\right\}$ of external $m$-contours in $V$ as $\left\{X_{\alpha}^{m}\right\} \cup\left\{Z_{\alpha}^{m}\right\}$, where $\left\{Z_{\alpha}^{m}\right\}$ denotes the small contours in $\left\{Y_{\alpha}^{m}\right\}$ and $\left\{X_{\alpha}^{m}\right\}$ the large contours in $\left\{Y_{\alpha}^{m}\right\}$. Note that for fixed $X_{\alpha}^{m}$ 's, the sum over $\left\{Z_{\alpha}^{m}\right\}$ goes over all sets of mutually external small contours in Ext $=V \backslash \bigcup_{\alpha}\left(X_{\alpha} \cup\right.$ Int $\left.X_{\alpha}\right)$, see Fig. 3.1. We therefore obtain, resuming the small contours and using the relation (2.6) a second time (this time for $Z_{m}^{\text {small }}$ ),

$$
\frac{Z_{m}(V)}{Z_{q}(V)}=\frac{1}{Z_{q}(V)} \sum_{\left\{X_{\alpha}^{m}\right\}_{\mathrm{ext}}} Z_{m}^{\mathrm{small}}(\mathrm{Ext}) \prod_{\alpha}\left[\rho\left(X_{\alpha}^{m}\right) e^{-e_{m}\left|X_{\alpha}^{m}\right|} \prod_{m^{\prime}} Z_{m^{\prime}}\left(\mathrm{Int}_{m^{\prime}} X_{\alpha}^{m}\right)\right] \text {. }
$$

Dividing by $\prod_{\alpha, m^{\prime}} Z_{q}\left(\operatorname{Int}_{m^{\prime}} X_{\alpha}^{m}\right)$ and multiplying it back again we obtain

$$
\frac{Z_{m}(V)}{Z_{q}(V)}=\sum_{\left\{X_{\alpha}^{m}\right\} \text { ext }} \frac{Z_{m}^{\text {small }}(\mathrm{Ext}) Z_{q}(\mathrm{Int}) e^{-e_{m} \sum_{\alpha}\left|X_{\alpha}^{m}\right|}}{Z_{q}(V)} \prod_{\alpha}\left[\rho\left(X_{\alpha}^{m}\right) \prod_{m^{\prime}} \frac{Z_{m^{\prime}}\left(\mathrm{Int}_{m^{\prime}} X_{\alpha}^{m}\right)}{Z_{q}\left(\mathrm{Int}_{m^{\prime}} X_{\alpha}^{m}\right)}\right]
$$

where the sum goes over sets of mutually external large contours in $V$ and Int $=\bigcup_{\alpha} \operatorname{Int} X_{\alpha}^{m}$.

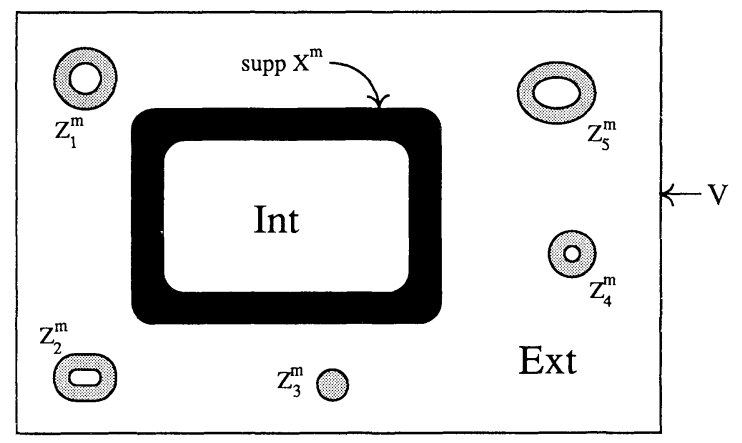

Fig. 3.1 Large and small external contours 
Note that all $q$-contours in $V$ and all small $m$-countours in $V$ are stable by the inductive assumptions i) and iii), respectively. Therefore the various partition functions in the first factor on the right-hand side of (3.13) are equal to the corresponding truncated partition functions, which can be controlled by convergent cluster expansions. Extracting the volume dependence and bounding the boundary terms, we find

$$
\left|\frac{Z_{m}^{\text {small }}(\operatorname{Ext}) Z_{q}(\operatorname{Int}) e^{-\sum_{\alpha} e_{m}\left|X_{\alpha}^{m}\right|}}{Z_{q}(V)}\right| \leqq e^{-\operatorname{Re}\left\{h_{m}^{\text {small }}|\operatorname{Ext}|+e_{m} \sum_{\alpha}\left|X_{\alpha}^{m}\right|-h_{q}|V \backslash \mathrm{Int}|\right\}} e^{O(\delta)\{|\partial \mathrm{Ex}|+|\partial \mathrm{Int}|+|\partial V|\}},
$$

where $h_{m}^{\text {small }}$ is the free energy obtained from $Z_{m}^{\text {small }}$. Using the fact that $\left|h_{m}^{\text {small }}-e_{m}\right| \leqq$ $O(\varepsilon)$ and using (3.7) to bound $|\partial \mathrm{Ext}|+|\partial \operatorname{Int}|$ by $|\partial V|+2 d \sum_{\alpha}\left|X_{\alpha}^{m}\right|$, we find that

$$
\left|\frac{Z_{m}^{\text {small }}(\mathrm{Ext}) Z_{q}(\mathrm{Int}) e^{-e_{m} \sum_{\alpha}\left|X_{\alpha}^{m}\right|}}{Z_{q}(V)}\right| \leqq e^{\operatorname{Re}\left(h_{m}^{\mathrm{small}}-h_{q}|| V \backslash \mathrm{Int}|+O(\varepsilon)| \partial V \mid\right.} \prod_{\alpha} e^{\left|X_{\alpha}^{m}\right|}
$$

Combining this bound with (3.13), the a priori estimate on $\rho$ and the inductive assumption (3.11) we get

$$
\left|\frac{Z_{m}(V)}{Z_{q}(V)}\right| \leqq e^{O(\varepsilon)|\partial V|} \sum_{\left\{X_{m}^{\alpha}\right\}_{\text {ext }}} e^{-\operatorname{Re}\left(h_{m}^{\text {small }}-h_{q}|| \backslash \backslash \backslash \mathrm{ntt} \mid\right.} \prod_{\alpha} e^{-(\tau-2 d-1) \mid X_{\alpha}^{m} !} .
$$

At this point we need a technical lemma proved in [Z1] (for the convenience of the reader, we give the proof below).

Lemma 3.2. Consider an arbitrary contour functional $\widetilde{K}\left(Y^{m}\right) \geqq 0$, and let $\tilde{Z}$ be the partition function

$$
\tilde{Z}(V)=\sum_{\left\{Y_{\alpha}^{m}\right\}} \prod_{\alpha}\left[\tilde{K}\left(Y_{\alpha}^{m}\right) e^{2 d\left|Y_{\alpha}^{m}\right|}\right] .
$$

Let $\tilde{s}$ be the corresponding free energy and assume that $\tilde{K}\left(Y^{m}\right) \leqq \widetilde{\varepsilon}^{Y^{m_{1}}}$, where $\tilde{\varepsilon}$ is small (depending on $N$ and $d$ ). Then for any $\tilde{a} \geqq-\tilde{s}$ the following bound is true

$$
\sum_{\left\{Y_{\alpha}^{m}\right\} \times x t} e^{-\tilde{a} \mid V \backslash \mathrm{nnt}} \prod_{\alpha} \tilde{K}\left(Y_{\alpha}^{m}\right) \leqq e^{o(\tilde{\imath})|\alpha V|}
$$

where the sum goes over sets of mutually external m-contours in $V$.

To apply this lemma we put

$$
\begin{aligned}
& \tilde{\varepsilon}=e^{-(\tau-2 d-1)}, \\
& \tilde{a}=\operatorname{Re}\left(h_{m}^{\text {small }}-h_{q}\right)=a_{m}+\operatorname{Re}\left(h_{m}^{\text {small }}-h_{m}\right),
\end{aligned}
$$

and

$$
\tilde{K}\left(Y^{m}\right)=\left\{\begin{array}{lll}
\tilde{\varepsilon}^{\left|Y^{m}\right|} & \text { if } \quad Y^{m} \text { is large } \\
0 & \text { if } \quad Y^{m} \text { is small. }
\end{array}\right.
$$

For $\tau$ large enough (depending on $d$ and $N$ ) the Mayer expansion for $\log \tilde{Z}(V)$ is convergent. Using the fact that it only contains large polymers (which implies $\left|Y^{m}\right| \geqq 1 / a_{m}$ for each polymer contributing to $\log \widetilde{Z}(V)$ ) one obtains that, for $\tau$ large 
(depending on $N$ and $d$ ) $0 \leqq-\tilde{s} \leqq e^{-\tau / 2 a_{m}}$. On the other hand, again for $\tau$ large,

$$
\left|h_{m}^{\text {small }}-h_{m}\right| \leqq e^{-\tau / 2 a_{m}} \text {, }
$$

which together with the above bound, implies that

$$
\tilde{a}+\tilde{s} \geqq a_{m}-2 e^{-\tau / 2 a_{m}} \text {. }
$$

Bounding

$$
e^{-\tau / 2 a_{m}} \leqq \frac{2 a_{m}}{\tau},
$$

we find that $\tilde{a}+\tilde{s} \geqq 0$ provided $\tau$ is large enough. This concludes the proof of ii).

Proof of iii) for diam $V=k$. By the inductive assumption all $m$-contours in $V$ are stable. Therefore $Z_{m}(V)=Z_{m}^{\prime}(V) \neq 0$. The inequality (3.12) follows immediately from (3.10), (3.11), and the fact that

$$
a_{m}|V| \leqq a_{m} \operatorname{diam} V|\partial V| \leqq|\partial V| .
$$

Proof of Lemma 3.2. $\tilde{Z}$ is the partition function of a polymer model with activities $K^{*}\left(Y^{m}\right)=\widetilde{K}\left(Y^{m}\right) e^{2 d\left|Y^{m}\right|}$. For $\tilde{\varepsilon}$ small enough (depending only on $d$ and $\left.N\right), \tilde{Z}$ can be controlled by a convergent expansion and

$$
|\log \tilde{Z}(W)+\tilde{s}| W|| \leqq O(\tilde{\varepsilon})|\partial W| \leqq|\partial W| .
$$

Putting $W=\operatorname{Int} Y_{\alpha}$ and using (3.7) together with the assumption $\tilde{a} \geqq-\tilde{s}$, we get

$$
\begin{aligned}
\sum_{\left\{Y_{\alpha}^{m}\right\}_{\text {ext }}} e^{-\tilde{a} \mid V \backslash \text { Int }} \prod_{\alpha}\left|\tilde{K}\left(Y_{\alpha}^{m}\right)\right| & \leqq e^{\tilde{s}|V|} \sum_{\left\{Y_{\gamma}^{m}\right\rangle_{\text {ext }}} \prod_{\alpha}\left|\tilde{K}\left(Y_{\alpha}^{m}\right)\right| e^{2 d\left|Y_{\alpha}^{m}\right|} \tilde{Z}\left(\text { Int } Y_{\alpha}^{m}\right) \\
& =e^{\tilde{s}|V|} \tilde{Z}(V) \leqq e^{O(\tilde{\varepsilon})|\partial V|}
\end{aligned}
$$

Assume now that for $\mu=\mu^{(0)}$ all the energies $e_{q}$ have the same real part. Our goal is to show that for some $\mu^{*}$ near $\mu^{(0)}$ all $a_{q}$ are zero, i.e., all b.c. are stable; more generally we will construct curves $\mu_{q}(t)$ going out of $\mu^{*}$, on which only $q$ is unstable, surfaces $\mu_{q \bar{q}}(t, s)$ on which $q, \bar{q}$ are unstable, etc. In order for this construction to work, some control on how quantities change with $\mu$ is needed. Our starting point is the following.

Theorem 3.3. Assume that in some open set $\mathscr{V}^{(0)} \subseteq \mathbb{R}^{N-1}, e_{m}$ and $\rho(Y)$ are continuously differentiable functions of $\mu$, and that $|\rho(Y)| \leqq e^{-\tau|Y|},\left|d \rho(Y) / d \mu_{i}\right| \leqq e^{-\tau|Y|}$, and $\left|d e_{m} / d \mu_{i}\right| \leqq 1$ for $\mu \in \mathscr{V}^{(0)}$. Then, for $\mu_{0} \in \mathscr{V}^{(0)}$ and $\tau \geqq \tau_{0}$, where $\tau_{0}$ is some constant depending only on $d$ and $N$, the following statements hold.

i) If $a_{q}\left(\mu_{0}\right)=0$ and $a_{m}\left(\mu_{0}\right) \operatorname{diam} V \leqq 1$, then

$$
\left|\frac{d}{d \mu_{i}} \frac{Z_{q}(V)}{Z_{m}(V)}\right|_{\mu=\mu_{0}} \leqq 4|V| e^{a_{m}\left(\mu_{0}\right)|V|+|\partial V|} .
$$

ii) For $a_{q}\left(\mu_{0}\right)=0$ and all $m \in Q=\{1, \ldots, N\}$,

$$
\left|\frac{d}{d \mu_{i}} \frac{Z_{m}(V)}{Z_{q}(V)}\right|_{\mu=\mu_{0}} \leqq 4|V| e^{|\partial V|} .
$$


iii) For $a_{m}\left(\mu_{0}\right) \operatorname{diam} V \leqq 1$ and $\forall \tilde{m} \in Q$,

$$
\left|\frac{d}{d \mu_{i}} \frac{Z_{\tilde{m}}(V)}{Z_{m}(V)}\right|_{\mu=\mu_{0}} \leqq 8|V| e^{3|\partial V|}
$$

Proof. We again proceed by induction. So we assume that $\operatorname{diam} V=k$ and that i), ii) and iii) have already been proven for all $W$ with $\operatorname{diam} W \leqq k-1$. In a preliminary step we show that for some open neighborhood $\mathscr{V}=\mathscr{V}(V)$ of $\mu_{0}$, all $m \in Q=\{1, \ldots, N\}$ and all contours $Y^{m}$ in $V$, the condition

$$
a_{m}\left(\mu_{0}\right) \operatorname{diam} Y^{m} \leqq 1
$$

implies that

ג) $Y^{m}$ is stable if $\mu \in \mathscr{V}(V)$,

B) $K\left(Y^{m}\right)$ is continuously differentiable in $\mathscr{V}(V)$,

$\gamma)$ For $\mu \in \mathscr{V}(V)$, the following bounds holds:

$$
\left|\frac{d}{d \mu_{i}} K\left(Y^{m}\right)\right| \leqq e^{-(\tau-16 d) \mid Y^{m}} .
$$

Proof of $(\alpha)-(\gamma)$. We use the fact that $Z_{\tilde{m}}(W)$ is defined as a finite sum of $C^{1}$ functions (see Equ. (2.2)). Therefore $Z_{\tilde{m}}(W) \in C^{1}\left(\mathscr{V}^{(0)}\right)$ for all $\tilde{m} \in Q$ and all finite $W$. Using Theorem $3.1 \mathrm{iii)}$, the inductive assumption (3.17) implies that

$$
Z_{m}\left(\operatorname{Int}_{\tilde{m}} Y^{m}\right) \neq 0 \quad \forall \tilde{m} \in Q
$$

at $\mu=\mu_{0}$. By continuity (3.19) is true in an open neighborhood $\tilde{\mathscr{V}}_{1}=\tilde{\mathscr{V}}_{1}\left(Y^{m}\right)$ of $\mu_{0}$. Therefore,

$$
f_{\tilde{m}}\left(Y^{m}\right)=\frac{Z_{\tilde{m}}\left(\operatorname{Int}_{\tilde{m}} Y^{m}\right)}{Z_{m}\left(\operatorname{Int}_{\tilde{m}} Y^{m}\right)} \in C^{1}\left(\tilde{\mathscr{V}}_{1}\left(Y^{m}\right)\right)
$$

for all $\tilde{m} \in Q$, which shows in particular that $K\left(Y^{m}\right)$ is continuously differentiable in $\tilde{\mathscr{V}}_{1}\left(Y^{m}\right)$.

By Theorem 3.1 iii),

$$
\left|f_{\tilde{m}}\left(Y^{m}\right)\right| \leqq \exp \left(3\left|\partial \operatorname{Int}_{\tilde{m}} Y^{m}\right|\right)
$$

at $\mu=\mu_{0}$. By continuity $Y^{m}$ is stable in a neighborhood $\tilde{\mathscr{V}}_{2}\left(Y^{m}\right)$, i.e.

$$
\left|f_{\tilde{m}}\left(Y^{m}\right)\right| \leqq \exp \left(4\left|\partial \operatorname{Int}_{\tilde{m}} Y^{m}\right|\right) \text {. }
$$

By the inductive assumption, and the continuity of $\partial f_{\tilde{m}} / \partial \mu_{i}$ in $\tilde{\mathscr{V}}_{1}\left(Y^{m}\right)$,

$$
\left|\frac{d}{d \mu_{i}} f_{\tilde{m}}\left(Y^{m}\right)\right| \leqq 8\left|\operatorname{Int}_{\tilde{m}} Y^{m}\right| \exp \left(4\left|\partial \operatorname{Int}_{\tilde{m}} Y^{m}\right|\right)
$$

in an open neighborhood $\tilde{\mathscr{V}}_{3}\left(Y^{m}\right)$ of $\mu_{0}$. For $\mu \in \tilde{\mathscr{V}}\left(Y^{m}\right)=\tilde{\mathscr{V}}_{1} \cap \tilde{\mathscr{V}}_{2} \cap \tilde{\mathscr{V}}_{3}$ we obtain

$$
\left|\frac{d}{d \mu_{i}} K\left(Y^{m}\right)\right|=\left|\frac{d}{d \mu_{i}}\left(\rho\left(Y^{m}\right) \prod_{\tilde{m}} f_{\tilde{m}}\left(Y^{m}\right)\right)\right| \leqq\left[1+8\left|\operatorname{Int} Y^{m}\right|\right] e^{-\tau\left|Y^{m}\right|+4 \mid \partial \operatorname{Int} Y^{m}} .
$$


Using (3.7) and the inequality

$$
1+8\left|\operatorname{Int} Y^{m}\right| \leqq 1+8\left|\partial \operatorname{Int} Y^{m}\right|^{2} \leqq \exp 8 d\left|Y^{m}\right|
$$

we obtain (3.18) for $\mu \in \tilde{\mathscr{V}}\left(Y^{m}\right)$. Since $V$ contains only finitely many contours, $(\alpha)$ to $(\gamma)$ are proven for

$$
\mathscr{V}(V)=\bigcap_{m} \bigcap_{Y^{m} \text { in } V} \mathscr{V}\left(Y^{m}\right)
$$

After these preliminaries, the proof of i) to iii) is essentially the same as that of Theorem 3.1. We start with the

Proof of i) for diam $V=k$. We rewrite

$$
\frac{d}{d \mu_{i}} \frac{Z_{q}(V)}{Z_{m}(V)}=\frac{Z_{q}(V)}{Z_{m}(V)} \frac{d}{d \mu_{i}}\left[\log Z_{q}(V)-\log Z_{m}(V)\right]
$$

and use Theorem 3.1 to bound $Z_{q} / Z_{m}$. To bound $d \log Z_{m}(V) / d \mu_{i}$, we note that $Z_{m}(V)=Z_{m}^{\prime}(V)$ for $\mu \in \mathscr{V}(V)$. Therefore the derivative of $\log Z_{m}$ can be bounded using the Mayer expansion for $\log Z_{m}-|V| e_{m}$ together with the bound (3.18). One obtains

$$
\left|\frac{d}{d \mu_{i}} \log Z_{m}\right| \leqq|V| \frac{d e_{m}}{d \mu_{i}}|+| V\left|O\left(e^{-\tau}\right) \leqq\right| V \mid\left(1+O\left(e^{-\tau}\right)\right) .
$$

Since trivially $a_{q}\left(\mu_{0}\right)$ diam $V=0 \leqq 1$, the same argument applies for the derivative of $\log Z_{q}(V)$. Therefore,

$$
\left|\frac{d}{d \mu_{i}} \frac{Z_{q}(V)}{Z_{m}(V)}\right| \leqq\left|\frac{Z_{q}(V)}{Z_{m}(V)}\right|\left[|V|\left(2+O\left(e^{-\tau}\right)\right)\right] \leqq\left|\frac{Z_{q}(V)}{Z_{m}(V)}\right| 4|V|
$$

if $\tau$ is large enough. This bound, together with Theorem 3.1, implies i).

Proof of ii) for diam $V=k$. We use Eq. (3.13). By the inductive assumption, Theorem 3.1 and the a priori bound on the derivative of $\rho(Y)$,

$$
\begin{aligned}
\left|\frac{d}{d \mu_{i}} \prod_{\alpha}\left[\rho\left(X_{\alpha}^{m}\right) \prod_{m^{\prime}} \frac{Z_{m^{\prime}}\left(\operatorname{Int}_{m^{\prime}} X_{\alpha}^{m}\right)}{Z_{q}\left(\operatorname{Int}_{m^{\prime}} X_{\alpha}^{m}\right)}\right]\right|_{\mu=\mu_{0}} \leqq & \exp \left[\sum_{\alpha}\left(\sum_{m^{\prime}}\left|\partial \operatorname{Int}_{m^{\prime}} X_{\alpha}^{m}\right|-\tau\left|X_{\alpha}^{m}\right|\right)\right] \\
& \cdot \sum_{\alpha}\left[1+4 \sum_{\tilde{m}}\left|\operatorname{Int}_{\tilde{m}} X_{\alpha}^{m}\right|\right] \\
\leqq & \sum_{\alpha}\left[\left|X_{\alpha}^{m}\right|+4\left|\operatorname{Int} X_{\alpha}^{m}\right|\right] \prod_{\alpha} e^{-(\tau-2 d)\left|X_{\alpha}^{m}\right|}
\end{aligned}
$$

where we used (3.7) in the last step. The derivative of the first factor in (3.13) is bounded as in the proof of i). The relevant estimates are

$$
\mid \frac{d}{d \mu_{i}} \log Z_{m}^{\text {small }}(\text { Ext })|\leqq[1+O(\varepsilon)]| \mathrm{Ext} \mid,
$$




$$
\begin{aligned}
\left|\frac{d}{d \mu_{i}} \log \frac{Z_{q}(V)}{Z_{q}(\operatorname{Int})}\right| & \leqq[1+O(\varepsilon)]|V \backslash \operatorname{Int}|, \\
\left|\frac{d}{d \mu_{i}} \sum_{\alpha} e_{m}\right| X_{\alpha}^{m}|| & \leqq \sum_{\alpha}\left|X_{\alpha}^{m}\right|,
\end{aligned}
$$

and (3.14). Putting everything together we obtain

$$
\begin{aligned}
\left|\frac{\partial}{\partial \mu_{i}} \frac{Z_{m}(V)}{Z_{q}(V)}\right| \leqq & e^{O(\varepsilon)|\partial V|} \sum_{\left\{X_{\alpha}^{m}\right\rangle \mathrm{cxt}} e^{-\operatorname{Re}\left(h_{m}^{\mathrm{smal1}}-h_{q}\right)|V \backslash \mathrm{Int}|} \prod_{\alpha} e^{-(\tau-2 d-1)\left|X_{\alpha}^{m}\right|} \\
& \cdot\left\{(1+O(\varepsilon))|\operatorname{Ext}|+(1+O(\varepsilon))|V \backslash \operatorname{Int}|+\sum_{\alpha}\left(2\left|X_{\alpha}^{m}\right|+4\left|\operatorname{Int} X_{\alpha}^{m}\right|\right)\right\} .
\end{aligned}
$$

Bounding the curly bracket by $4|V|$ (use that $\mid$ Ext $\left|+\sum_{\alpha}\right| X_{\alpha}^{m}|=| V \backslash$ Int $\mid$ ) and using Lemma 3.2 as in the proof of Theorem 3.1 we get ii).

Proof of iii) for diam $V=k$. iii) is an immediate consequence of i), ii), Theorem 3.1 and the fact that for any $q$ with $a_{q}\left(\mu_{0}\right)=0$,

$$
\frac{d}{d \mu_{i}} \frac{Z_{\tilde{m}}(V)}{Z_{m}(V)}=\frac{d}{d \mu_{i}}\left(\frac{Z_{\tilde{m}}(V)}{Z_{q}(V)} \frac{Z_{q}(V)}{Z_{m}(V)}\right)
$$

(use that $2|\partial V|+a_{m}\left(\mu_{0}\right)|V| \leqq 3|\partial V|$ if $a_{m}\left(\mu_{0}\right)$ diam $V \leqq 1$ ).

Corollary 3.4. Let $\mathscr{V}$ be an open set $\mathscr{V} \subseteq \mathbb{R}^{N-1}$ such that $e_{m}$ and $\rho(Y)$ are continuously differentiable functions of $\mu$ in $\mathscr{V}$, and such that $|\rho(Y)| \leqq e^{-\tau|Y|},\left|d \rho(Y) / d \mu_{i}\right| \leqq e^{-\tau|Y|}$, $\left|d e_{m} / d \mu_{i}\right| \leqq 1$ for $\mu \in \mathscr{V}$. Assume that for a given $m \in Q$,

$$
a_{m}(\mu) \operatorname{diam} Y^{m} \leqq 1, \quad \forall \mu \in \mathscr{V} .
$$

Then $K\left(Y^{m}\right)$ is in $C^{1}(\mathscr{V})$ and

$$
\begin{aligned}
\left|K\left(Y^{m}\right)\right| & \leqq e^{-(\tau-8 d)\left|Y^{m}\right|} \\
\left|\frac{d}{d \mu_{i}} K\left(Y^{m}\right)\right| & \simeq e^{-(\tau-16 d)\left|Y^{m}\right|} .
\end{aligned}
$$

Proof. Fix $Y^{m}$ and $\mu$ in such a way that

$$
a_{m}(\mu) \operatorname{diam} Y^{m} \leqq 1
$$

As shown in the preliminary step of the proof of Theorem 3.3, this implies that $K\left(Y^{m}\right)$ is continuously differentiable in a neighborhood of $\mu$, together with the bounds (3.23) and (3.24). Since the assumption (3.25) is fulfilled in all of $\mathscr{V}$, the corollary is proven.

\section{Application to $\boldsymbol{P}(\phi)_{2}$ Models}

We show here how $P(\phi)_{2}$ models with multiple minima can be put into the framework of this paper. We work with the models considered in [I]; a polynomial $\mathscr{P}$ is chosen which has any number of minima but which has positive curvature, 
or mass-squared, at each minimum. The polynomial is scaled into $\lambda^{-2} \mathscr{P}(\lambda \phi)$, with $\lambda$ small, which leads to a weak coupling, mean field regime with large barriers between the minima. The coefficients in $\mathscr{P}$ depend smoothly on parameters $\mu$, and we fix $\lambda$ when mapping out the phase diagram in $\mu$-space. A detailed description of the setup can be found in Sect. 2.1 of [I]. A degeneracy-removing condition is assumed for the values of $\mathscr{P}$ at its minima.

This paper provides a considerably simpler method of analyzing the phase structure of these models. In addition, it allows us to work with complex polynomials - the method is insensitive to the phases of the polymer activities. Thus starting with a real interaction $\lambda^{-2} \mathscr{P}(\lambda \phi)$ as above, we add an imaginary one $i \mathscr{Q}(\lambda \phi)$, where $\mathscr{Q}$ is any real polynomial. We omit the prefactor $\lambda^{-2}$ because we wish to treat linear and quadratic terms perturbatively. In this case there are no new issues in the cluster expansion estimates (which we borrow from [I]. More generally one could imagine "rotating the contours of $\phi$-integration" to follow the critical points of the interaction as they move off the real axis. We do not pursue this possibility here.

We recall a few notations from [I]. Let us define $P(\phi)=\lambda^{-2} \mathscr{P}(\lambda \phi)+i \mathscr{Q}(\lambda \phi)$. The basic vacuum energy estimates in the vicinity of each minimum are governed by $\operatorname{Re} P$. In particular, we use masses $m_{q}$ computed from the curvature of $\operatorname{Re} P$ at its minima $\xi_{q}$. We use the approximation $P\left(\xi_{q}+\phi\right) \approx \frac{1}{2} m_{q}^{2} \phi^{2}$ near each minimum and expand in terms of the corresponding Gaussian measure. We put $E_{c}^{q}=P\left(\xi_{q}\right)$, which is the dominant term in the vacuum energy estimates, with real part proportional to $\lambda^{-2}$. The complex part can be preserved in the estimates since it is a constant independent of $\phi$. After $E_{c}^{q}$ there is an $O(1)$ contribution to the vacuum energies arising from the differing values of $m_{q}$. The shifted energy is denoted $E_{w}^{q}$.

The $P(\phi)_{2}$ expectation in a volume $\Lambda$ with boundary condition $q$ is defined as

$$
\langle\cdot\rangle_{\Lambda, q}=\frac{1}{N} \int \cdot \exp \left(\int_{\Lambda}\left[: P(\phi(x)):-\frac{1}{2} m_{q}^{2}:\left(\phi(x)-\xi_{q}\right)^{2}:\right] d x\right) d \mu_{m_{q}^{2}}\left(\phi-\xi_{q}\right),
$$

where $d \mu_{m_{q}^{2}}$ is the Gaussian measure with covariance $\left(-\Delta+m_{q}^{2}\right)^{-1}$. We prove the following theorem describing the phase diagram of this system.

Theorem 4.1. Given real polynomials $\mathscr{P}, \mathscr{Q}$ as above, depending on $\left\{\mu_{1}, \ldots, \mu_{N-1}\right\}$, let $P(\phi)=\lambda^{-2} \mathscr{P}(\lambda \phi)+i \mathscr{Q}(\lambda \phi)$ and let $\lambda$ be sufficiently small. There exists a continuous map from a neighborhood of the origin in the boundary of the positive octant in $\mathbb{R}^{N}$ onto a neighborhood of the origin in parameter space $\mathbb{R}^{N-1}$. The map is differentiable on each $j$-dimensional face, $1 \leqq j \leqq N-1$. If $\mu$ is the image of a point whose $q^{\text {th }}$ coordinate is zero, then the $q^{\text {th }}$ phase is stable. At $\mu$, the infinite volume limit of the Schwinger functions $\left\langle\phi\left(x_{1}\right) \cdots \phi\left(x_{n}\right)\right\rangle_{\Lambda, q}$ exists with exponential clustering and asymptotically of the perturbation theory in $\lambda$ around $\phi=\xi_{q}$. There are $\left(\begin{array}{l}n \\ K\end{array}\right)$ hypersurfaces of codimension $k-1$ in parameter space at which $k$ distinct phase coexist.

The cluster expansion of [I] used squares of side length $l \gg 1$ in the decoupling, so that each polymer is a union of such square. The larger $l$ is, the stronger the decay in the number of squares in a polymer. Each $l$-square is a unit square for 
the purposes of Sects. 2,3,5. The coupling $\lambda$ has to be chosen small enough, depending on $l$. The partition function for the $q^{\text {th }}$ phase in one of these squares is denoted $Z_{\Delta^{q}}$. Here the field is encouraged to lie near $\xi_{q}$ by factors inserted by hand into the measure. (These factors are a part of a partition of unity employed throughout the expansion.) We have a bound

$$
\left|Z_{\Delta^{q}} e^{l^{2} E_{w}^{q}}\right|^{ \pm 1} \leqq e^{a \lambda l^{2}}
$$

see Proposition 2.5.2 of [I]. Also, $\mu$-derivative of $\log \left(Z_{\Delta^{q}} e^{l^{2} E_{w}^{2}}\right)$ are of order $\lambda^{1 / 2}$ or so, so each $\log Z_{\Delta^{q}}$ follows closely the behavior of $E_{w}^{q} l^{2}$.

It is simplest to describe the expansion in the form where powers of $Z_{\Delta^{q}}$ have been factored out of the clusters making up the expansion. Consider the partition function $Z_{m}(V)(=Z(\mathbb{V})$, with $\mathbb{V}=(V, m)$ in the notation of $[\mathrm{I}])$. Let $\Sigma$ be an association of a phase $q$ to each unit square of $V$, and let $R_{q}^{\Sigma}$ be the union of the squares in the phase $m$. We denote by $\left|R_{q}^{\Sigma}\right|$ the area of this region, in units of $l^{2}$. The expansion is

$$
Z_{m}(V)=\sum_{\Sigma} \sum_{\left\{\mathbb{V}_{k}\right\} \text { compatible with } \Sigma} \prod_{k} \tilde{\rho}\left(\mathbb{\mho}_{k}\right) \prod_{q} Z_{\Delta}^{\mid \mathbb{R}_{q}^{\Sigma}} .
$$

Here $\mathbb{Y}$ specifies a region $Y$ as well as $\Sigma \uparrow Y$, so the clusters of $[\mathrm{I}]$ contain more structure than is needed for our present analysis. We will sum over the unnecessary structure shortly. The cluster functional $\tilde{\rho}(\mathbb{Y})$ is taken directly from $[I]$ and obeys a bound

$$
|\tilde{\rho}(\mathbb{Y})| \leqq \lambda^{1 / 2} e^{-5 / 2 \tau_{1}|| Y \mid} e^{-2 \tau_{2} \iota^{-2}\left|\Sigma_{\curlyvee}\right|},
$$

where $\tau_{1}, \tau_{2}$ are positive constants, $|\mathbb{Y}|$ is the area of $Y$ in units of $l^{2}$, and $\left|\Sigma_{\gamma}\right|$ is the length of the "contour" separating subsets of $Y$ with constant phase [see [I], (2.5.11)]. Also, $\tilde{\rho}(\mathbb{Y})=1$ if $|\mathbb{Y}|=1$, since the entire contribution from such $\mathbb{Y}$ is in the $Z_{\Delta^{q}}$ factors. Thus the regions $\mathbb{Y}_{k}$ fill all of $V$. The expansion (4.2) follows, with a little bookkeeping, from [I], (2.4.22) and (2.5.12).

To put (4.2) into a form like (2.2), we need to sum over all Y's compatible with a given region $Y$ and with some assignment $q(\cdot)$ of phases for each boundary component of $Y$. This will not lead to exponentially decaying activities as in (2.1) unless we take care of the very high energy phases properly. Define

$$
e_{q}=-\log Z_{\Delta^{q}}
$$

By (4.1) and the discussion there, we see that $e_{q}$ is close to $l^{2} E_{c}^{q}$ (also the $\mu$-derivatives are close). Therefore for small $\lambda$ the degeneracy-removing condition (2.3) holds for $\left\{e_{q}\right\}$ if it holds for $E_{c}^{q}$. (To normalize the matrix $\partial e_{q} / \partial \mu_{i}$ properly for (2.4) we would rescale $\left\{\mu_{i}\right\}$ by a factor $O\left(\lambda^{-2} l^{2}\right)$.) Let us assume the energies $e_{q}$ are increasing in $q$, i.e. $e_{1} \leqq e_{2} \leqq \cdots \leqq e_{M}$. Then any phase $p$ with $e_{p} \geqq e_{1}+\frac{1}{2} \tau_{1} l$ is clearly out of contention for stability. (We shall see that corrections to the approximate free energies $e_{m}$ are $e^{-O\left(\tau_{1} l\right)}$.) Let $e_{1}, \ldots, e_{N}$ be the low energies, and $e_{N+1}, \ldots, e_{M}$ be the higher energies, with $N$ chosen so that $e_{N+1} \geqq e_{1}+\frac{1}{2} \tau_{1} l, e_{N} \leqq e_{1}+\tau_{1} l$, and $e_{N+1} \geqq e_{N}+(1 / 2 M) \tau_{1} l$. (To simplify the subsequent analysis we make the division at a point where a gap occurs.)

Now let $Y$ be a contour as in Sects. 2,3 (that is, a region, together with an 
assignment of boundary conditions $m \in\{1, \ldots, N\}$ to boundary components). We sum over all ways of paving $Y$ with collections of clusters $\left\{\mathbb{Y}_{\alpha}\right\}$ which could have arisen in the expansion (4.2) and which agree with the given boundary condition assignments. We restrict the sum to collections such that any part of a $\partial \Psi_{\alpha}$ not already present in $\partial Y$ has a high-energy boundary condition $p \in\{N+1, \ldots\}$. The point is to make all high-energy phases internal to the contour $Y$, so that they are not seen in subsequent analysis. Any loss of exponential decay in $\tilde{\rho}\left(\mathbb{Y}_{\alpha}\right)$, say from a single-square cluster $\left|\mathbb{Y}_{\alpha}\right|=1$, is made up by the smallness of $Z_{\Delta^{q}}$, since

$$
\left|Z_{\Delta^{p}}\right| \leqq e^{-e_{N}-\tau_{1} l / 2 M}
$$

for $p \geqq N+1$. Thus we define

$$
\rho(Y)=\sum_{\left\{\mathbb{V}_{\alpha}\right\}} \prod_{\alpha} \tilde{\rho}\left(\mathbb{Y}_{\alpha}\right) \prod_{q} Z_{\Delta^{q}}^{\left|R_{q}^{\Sigma}\right|} \prod_{m} e^{e_{m}\left|R_{m}\right|} .
$$

We use a convention that $q$ runs over all phases (low or high energy), while $m$ runs from 1 to $N$ and $p \geqq N+1$ labels the high energy phases. The regions $R_{q}^{\Sigma}$ and $R_{m}$ are what appear in expansions (4.2), (2.2), respectively, although they are restricted to the set $Y$. It should be evident that the desired form of the expansion (2.2) holds now with this prescription for $\rho(Y)$. We drop any $Y$ with $|Y|=1$ since in that case $\rho(Y)=1$.

We verify that $\rho(Y)$ decays exponentially as in (2.1). The decay of $\tilde{\rho}(\mathbb{Y})$ specified by (4.3) permits us to sum over $\Sigma$ in $Y$. The remaining combinatorics, including the sum over pavings of $Y$ by clusters $\mathbb{Y}_{\alpha}$, produce a factor $c^{|Y|}$ in the estimate on $\rho(Y)$. The other large factors arise from factors $Z_{\Delta^{m^{\prime}}} e^{-e_{m}}$, which can be as large as $e^{\tau_{1} l}$. Both of these effects are controlled by $e^{-5 / 2 \tau_{1} l|\vartheta|}$ wherever $|\mathbb{Y}|>1$. If $|\mathbb{Y}|=1$, then $\Sigma_{\curlyvee} \equiv p \geqq N+1$ and by (4.5) we have $Z_{\Delta^{p}} e^{-e_{m}} \leqq e^{-\tau_{1} l / 2 M}$, and we still have a good decay in $|Y|$. Thus (2.1) holds with $\tau=\tau_{1} l / 2 M$, which can be made as large as desired by increasing $l$ (and decreasing $\lambda$ ). The corresponding bound on $\partial \rho(Y) / \partial \mu_{i}$ follows in a similar fashion. The needed bound on $\partial \tilde{\rho} / \partial \mu_{i}$ is the same as (4.3) and is also proven in [I].

Having put the expansion in the form (2.2), the constructions of Sect. 3 lead to a determination of the stable phases and to a proof of ratio of the partition function bound

$$
\left|\frac{Z_{m}(V)}{Z_{q}(V)}\right| \leqq e^{|\partial V|},
$$

(see (3.11). The phase diagram is obtained by adjusting the parameters $\mu$ for coexistence-see Sect. 6. The intricate iteration of Chap. 3 of [I] is avoided. Also the "bounded-spin approximation" estimate is no longer needed - differentiability of the contour activities is sufficient for our analysis.

To obtain the infinite volume Schwinger functions for the stable phases and prove Theorem 4.1, it is necessary to have estimates on ratios of "constrained partition functions" in $V$. Here we mean that interiors of contours may not intersect $V^{c}$. Such estimates were used in Sect. 4.3 of [I] to control the cluster expansion for the Schwinger functions. (A similar procedure is used in [BW].) These 
constraints were built into the partition functions defined in Sect. 2. Thus the estimates of Sect. 3 apply already to the constrained case, and no additional arguments (cf. Sect. 4.2 of [I]) are needed.

\section{Periodic Partition Functions}

We apply the theory developed so far to partition functions on the $d$-dimensional torus $T$. We expect that these results will be useful in supersymmetric field theory models, especially the two-dimensional Wess-Zumino models considered in [JLW]. There an index theorem was proven which computes the value of the partition function in a periodic box (equivalently, the supertrace of $e^{-\beta H}$ ). The value is an integer index which is independent of the dimensions of the torus. When the integer is nonzero, the phase structure analysis developed here should imply that the vacuum energy of the model vanishes, and hence the usual criterion for absence of spontaneous supersymmetry breaking holds. Furthermore, when the vacuum energy is zero, some information on the number of stable phases can be extracted from the index - see Corollary 5.2 below. At present, these statements must be made contingent on a construction of a mean field expansion for the Wess-Zumino models (analogous to the one considered in Sect. 4 for $P(\phi)_{2}$ ). In view of the two-phase expansion for Yukawa [BG] and the single-phase expansion for the $N=1$ model [W], we believe that this is an accessible problem.

We define the periodic partition functions with additional factors $\sigma(m) \in \mathbb{C}$ multiplying the "measure" for configurations predominantly in the $m^{\text {th }}$ phase. These factors seem artificial from the point of view of the contour models but in fact arise naturally in the $N=1$ Wess-Zumino models. There we expect $\sigma(m)= \pm 1$, depending on the sign of the fermion mass. Here we assume only that $|\sigma(m)| \leqq 1$.

For simplicity, we let $T$ be a torus with length $L \in \mathbb{Z}$ in each direction. We seek upper and lower bounds of the form $e^{-f L^{d}}\left(A+e^{-B \tau L}\right)$, where the free energy $f$ and the constants $A, B$ are independent of $L$, and where $L$ is sufficiently large, depending on $\tau$ and on

$$
\tilde{a}=\min _{m: a_{m} \neq 0} a_{m} .
$$

We define $Z^{P}(T)$, the periodic partition function, by grouping together configurations which we wish to treat together in the estimates. This way we avoid having to introduce unneeded definitions. If the partition function so constructed seems unnatural, the reader should think about an example such as the Peierls expansion for the Ising model on a torus. It should be clear that the type of object we define is exactly what arises in that context.

Let $V$ be a subset of $T$, as usual built of unit cubes. We define restricted partition functions $Z_{m}^{\text {res }}(V)$ by considering only contours $Y$ in $V$ with $\operatorname{diam} Y<L / 2$. For such contours it is clear which component of $V \backslash Y$ is the exterior, and which ones are interior. Thus we can unambiguously say which are the external contours, and we require all of these to be $m$-contours. If $V \neq T$, then $\partial V \neq \emptyset$ and we are assuming $\partial V$ is at boundary condition $m$. The regions $R_{m^{\prime}}$ in (2.2) are defined as before, with the exterior region considered as part of $R_{m}$. It may happen that $\{Y\}=\emptyset$, in which case $R_{m}=V$. As always we assume $Y \cup$ Int $Y \subseteq V$. With these definitions we have 
as in (2.2),

$$
Z_{m}^{\mathrm{res}}(V)=\sum_{\left\{Y_{\alpha}\right\} \cdot \operatorname{diam} Y_{\alpha}<L / 2} \prod_{\alpha} \rho\left(Y_{\alpha}\right) \prod_{m^{\prime}} e^{-e_{m^{\prime}}\left|R_{m^{\prime}}\right|} .
$$

These contour activities are assumed to be equal to the corresponding ones in $\mathbb{R}^{d}$. Of course the alternative representations (2.6), (2.7) hold for $Z_{m}^{\text {res }}(V)$ as well, and for any $Y$ that appears, the diameter restriction is irrelevant in Int $Y$.

There remain the configurations where at least one contour is large, for example there may be contours wrapping around the torus. In this case, interior and exterior are ill-defined, so we slightly modify the construction in (2.2). We only need the full partition function $Z^{P}$ for the entire torus, so we restrict attention to this case. The remaining configurations are grouped into $Z^{\text {big }}(T)$. We join all large contours into a single contour $Y^{\text {big }}$, each connected component of which has diameter at least $L / 2$. As usual each component of $T \backslash Y^{\text {big }} \backslash \bigcup_{\alpha} Y_{\alpha}$ has a constant boundary condition. Each such component contributes to the appropriate $R_{m}$. Also, the small $m$-contours $Y_{\alpha}^{m}$ are part of $R_{m}$, as before. But $Y^{\text {big }}$ is not assigned to any $R_{m}$; any free energy associated to it is built into $\rho\left(Y^{\mathrm{big}}\right)$. With these definitions we put

$$
Z^{\text {big }}(T)=\sum_{Y^{\text {big }}} \rho\left(Y^{\text {big }}\right) \sum_{\left\{Y_{\alpha}\right\} \cdot \operatorname{diam} Y_{\alpha}<L / 2} \prod_{\alpha} \rho\left(Y_{\alpha}\right) \prod_{m} e^{-e_{m}\left|R_{m}\right|} .
$$

Finally, we define

$$
Z^{P}(T)=Z^{\mathrm{big}}(T)+\sum_{m=1}^{N} \sigma(m) Z_{m}^{\mathrm{res}}(T) .
$$

The absence of factors $\sigma(m)$ in $Z^{\text {big }}(T)$ means only that they are built into the activities there. Likewise, any departures from a simple factor $\sigma(m)$ in $Z_{m}^{\text {res }}(T)$ are built into the activities in that partition function. To state our main result on $Z^{P}(T)$ we let

$$
e_{0}=\min _{m} \operatorname{Re} e_{m}
$$

Theorem 5.1. Assume that $|\rho(Y)| \leqq e^{-\tau|Y|}$ for all $Y$ with $\operatorname{diam} Y<L / 2$ and that $\left|\rho\left(Y^{\mathrm{big}}\right)\right| e^{e_{0}\left|Y^{\mathrm{big}}\right|} \leqq e^{-\tau\left|Y^{\mathrm{big}}\right|}$ for all $Y^{\mathrm{big}}$. Then, for $\tau>\tau_{0}(d, N)$ and $L \geqq L_{0}(\tau, \tilde{a})$, the following bound holds:

$$
\left|Z^{P}(T)-\sum_{q} \sigma(q) e^{-h_{q} L^{d}}\right| \leqq e^{-B \tau L} e^{-f L^{d}} .
$$

Here $f=\min _{m} \operatorname{Re} h_{m}$ and $B>0$ is a fixed constant. Then sum in (5.5) goes only over stable q, i.e. $q$ such that $\operatorname{Re} h_{q}=f$.

The theorem can be written concisely in the case of real (not necessarily positive) activities and energies.

Corollary 5.2. Under the assumptions of Theorem 5.1, let us suppose that $\rho(Y)$, $\rho\left(Y^{\mathrm{big}}\right)$, and $e_{m}$ are all real, and that $\sigma(m)= \pm 1$. Then

$$
\lim _{L \rightarrow \infty} Z^{P}(T) e^{f L^{d}}=n_{+}-n_{-},
$$


where $n_{+}$is the number of stable phases with $\sigma(m)=1$ and $n_{-}$is the number with $\sigma(m)=-1$.

The physical content of these estimates is that each stable phase contributes equally to $Z^{P}(T)$, while the unstable phases are negligible for large $L$. Corrections to thermodynamic behavior are exponentially small in diam $T=L$. Note that we had to assume a different bound on $\left|\rho\left(Y^{\mathrm{big}}\right)\right|$ because the free energy associated to $Y^{\text {big }}$ was built into $\rho\left(Y^{\text {big }}\right)$.

Theorem 5.1 follows from the following three lemmas, which are proven in the remaining part of this section.

Lemma 5.3. There are constants $b_{1}>0, \tau_{1}<\infty$ such that the following statements hold for $\tau \geqq \tau_{1}$ and $q$ stable:

i)

$$
\left|\log Z_{q}^{\mathrm{res}}(V)+\right| V\left|h_{q}\right| \leqq|\partial V| e^{-b_{1} \tau}+e^{-b_{1} \tau L / 2}
$$

ii)

$$
\left|Z_{q}^{\mathrm{res}}(T) e^{h_{q} L^{d}}-1\right| \leqq e^{-b_{1} \tau L / 2} .
$$

Lemma 5.4. There are constants $b_{2}>0, \tau_{2}<\infty$, and $K_{2}<\infty$, such that for $\tau \geqq \tau_{2}$ the following statements hold:

i)

$$
\left|Z_{m}^{\mathrm{res}}(V) e^{f|V|}\right| \leqq \exp \left\{e^{-b_{1} \tau L / 2}+|\partial V|\right\},
$$

where $b_{1}$ is the constant from Lemma 3.1,

ii)

$$
\left|Z_{m}^{\mathrm{res}}(T) e^{f L^{d}}\right| \leqq e^{-b_{2} \tau L^{d-1}}
$$

provided $m$ is unstable and

$$
L \geqq K_{2} \tau / a_{m} .
$$

Lemma 5.5. There are constants $b_{3}>0, \tau_{3}<\infty$ such that

$$
\left|Z^{\mathrm{big}}(T) e^{f L^{d}}\right| \leqq e^{-b_{3} \tau L}
$$

provided $\tau \geqq \tau_{3}$.

Proof of Lemma 5.3. Comparing the convergent cluster expansions for $h_{q}$ and $\log Z_{q}^{\text {res }}(V)$ or $\log Z_{q}^{\text {res }}(T)$, respectively, one immediately obtains Lemma 5.3. The constant $b_{1}$ can be chosen arbitrarily close to 1 if $\tau_{1}$ is chosen large enough.

Proof of Lemma 5.4. We proceed as in the proof of Theorem 3.1 ii) in Sect. 3 to bound

$$
\begin{aligned}
\left|Z_{m}^{\mathrm{res}}(V) e^{f|V|}\right| \leqq & \exp \left\{|\partial V| e^{-b_{1} \tau}+e^{-b_{1} \tau L / 2}\right\} \\
& \sum_{\left\{X_{\alpha}^{m}\right\}_{\mathrm{ext}}}^{\prime} e^{\left(f-\operatorname{Re} h_{m}^{\text {small }}\right)|V \backslash \mathrm{Int}|} \prod_{\alpha} e^{-(\tau-2 d-1)\left|X_{\alpha}^{m}\right|},
\end{aligned}
$$

where the sum goes over sets $\left\{X_{\alpha}^{m}\right\}$ of mutually external $m$-contours which are all 
large (i.e. $a_{m} \operatorname{diam} X_{\alpha}^{m}>1$ ) and obey a bound diam $X_{\alpha}^{m}<L / 2$. Extracting a factor

$$
\max _{\left\{X_{\alpha}^{m}\right\rangle_{\text {ext }}} e^{-\left(a_{m} / 2\right)|V \backslash \mathrm{Int}|} \prod_{\alpha} e^{-(\tau / 2)\left|X_{\alpha}^{m}\right|}
$$

and continuing as before we obtain, for a suitable choice of $\tau_{2}<\infty$ and $\tau \geqq \tau_{2}$,

$$
\left|Z_{m}^{\mathrm{res}}(V) e^{f|V|}\right| \leqq \exp \left\{|\partial V|+e^{-b_{1} \tau L / 2}\right\} \max _{\left\{X_{\alpha}^{m}\right\}_{\text {ext }}} e^{-\left(a_{m} / 2\right)|V \backslash \mathrm{Int}|} \prod_{\alpha} e^{-(\tau / 2)\left|X_{\alpha}^{m}\right|} .
$$

Bounding the second factor on the right-hand side by 1 we obtain i). To prove ii) we set $V=T$ and use the isoperimetric inequality to bound

$$
|V \backslash \operatorname{Int}|=L^{d}-|\operatorname{Int}| \geqq L^{d}-K|\partial \operatorname{Int}|^{d /(d-1)},
$$

Where $K$ is a constant depending only on $d$. Bounding $|\partial \operatorname{Int}|$ by $2 d \Sigma\left|X_{\alpha}^{m}\right|$ we get

$$
\left|Z_{m}^{\mathrm{res}}(T) e^{f L^{d}}\right| \leqq \exp \left\{e^{-b_{1} \tau L / 2}\right\} \sup _{x \geqq 0} \exp \left(\frac{-\tau X}{2}-\max \left\{0, \frac{a_{m}}{2}\left(L^{d}-K(2 d X)^{d /(d-1)}\right)\right\}\right) .
$$

The supremum is obtained either for $X=0$ or for $L^{d}-K(2 d X)^{d /(d-1)}=0$ which proves that

$$
\left|Z_{m}^{\mathrm{res}}(T) e^{f L^{d}}\right| \leqq \exp \left\{e^{-b_{1} \tau L / 2}\right\} \max \left\{e^{-\left(a_{m} / 2\right) L^{d}}, e^{-(r / 2) K_{2} L^{d-1}}\right\},
$$

with $K_{2}=\left(2 d K^{(d-1) / d}\right)^{-1}$. This proves ii) with, for example, $b_{2}=K_{2} / 4$.

Proof of Lemma 5.5. Given a configuration $\left\{Y^{\mathrm{big}}, Y_{\alpha}\right\}$ contributing to $Z^{\mathrm{big}}(T)$ we let $V_{m}$ be the union of those components $C$ of $T \backslash Y^{\text {big }}$ which have boundary condition $m$. Resumming all small contours $Y_{\alpha}$ in the representation (5.3) for $Z^{\text {big }}(T)$ we obtain

$$
Z^{\mathrm{big}}(T)=\sum_{Y^{\mathrm{big}}} \rho\left(Y^{\mathrm{big}}\right) \prod_{m} Z_{m}^{\mathrm{res}}\left(V_{m}\right) .
$$

Using Lemma 5.3 i) and Lemma 5.4 i) together with the fact that $\left|e^{h_{q}\left|V_{q}\right|}\right|=e^{f\left|V_{q}\right|}$ if $q$ is stable, we bound

$$
\left|Z^{\mathrm{big}}(T) e^{f L^{d}}\right| \leqq \sum_{Y^{\mathrm{b} \mathbf{g}}}\left|\rho\left(Y^{\mathrm{big}}\right)\right| \exp \left[\left(f+2 d+e^{-b_{1} \tau L / 2}\right)\left|Y^{\mathrm{big}}\right|\right] .
$$

Next we use the bound (3.9) to conclude that for all $m=1, \ldots, N$,

This shows that

$$
f \leqq \operatorname{Re} h_{m} \leqq \operatorname{Re} e_{m}+O\left(e^{-\tau}\right) .
$$

$$
\left|\rho\left(Y^{\mathrm{big}}\right)\right| \exp \left[\left(f+2 d+e^{-b_{1} \tau L / 2}\right)\left|Y^{\mathrm{big}}\right|\right] \leqq \prod_{\alpha} e^{-(\tau-O(1))\left|X_{\alpha}\right|},
$$

where $\left\{X_{\alpha}\right\}$ are the (large) connected subcontours of $Y^{\text {big }}$. Bounding the sum over a connected contour $X$ with fixed size $|X|=s$ by $L^{d} K^{s}$, where $K$ is a constant depending only on $N$ and $d$, we get for $\tau$ large,

$$
\begin{aligned}
\left|Z^{\mathrm{big}}(T) e^{f L^{d}}\right| & \leqq \sum_{n=1}^{\infty} \frac{1}{n !}\left(L^{d} \sum_{s=L / 2}^{\infty} e^{-\tau s / 2}\right)^{n} \\
& =\exp \left\{L^{d} e^{-\tau L / 4}\left(1-e^{-\tau / 2}\right)^{-1}\right\}-1 \\
& \leqq e^{-\tau L / 8} .
\end{aligned}
$$




\section{The Phase Diagram}

We consider now the question of constructing the phase diagram of the contour model. First, we wish to find a point $\mu(0)$ in parameter space where $a_{q}=0$ for all $q \in Q$. From this point we construct $N$ curves at which all but one $a_{q}=0$, and so on to hypersurfaces of higher dimension at which various subsets of $Q$ satisfy $a_{q}=0$. As we have seen, the vanishing of $a_{q}$ is sufficient for the construction of the infinite volume state.

While this is a standard question in Pirogov-Sinai theory, the present situation with complex activities is more sutable because the $a_{q}$ are not necessarily continuous functions of the parameters. This appears to be an inevitable consequence of the presence of partition function zeros. Nevertheless, the closer $a_{q}$ is to zero, the better it can be approximated by a differentiable function. This allows us to construct differentiable curves at which some $a_{q}$ 's are zero. Higher order differentiability could be obtained with a corresponding strengthening of assumptions on the activities $\rho$.

The setup is as follows. We assume there is a bounded open set $\mathscr{U} \subset \mathbb{R}^{N-1}$ in which $\rho(Y), e_{m}$ are $C^{1}$ functions satisfying the bounds

$$
\begin{aligned}
|\rho(Y)|,\left|\frac{\partial}{\partial \mu_{i}} \rho(Y)\right| & \leqq e^{-\tau|Y|}, \\
\left|\frac{\partial e_{m}}{\partial \mu_{i}}\right| & \leqq 1
\end{aligned}
$$

This assumption allows us to use the basic estimates of Theorems 3.1 and 3.3. In addition, we assume a degeneracy-removing condition throughout $\mathscr{U}$. To be specific, with $i, m=1, \ldots, N-1$, we assume that the matrix $\partial \operatorname{Re}\left(e_{m}-e_{N}\right) / \partial \mu_{i}$ is invertible and its inverse is bounded (as an operator on $l_{\infty}(1, \ldots, N-1)$ ) by $M$ for some fixed $M<\infty$. We assume that $\mathscr{U}$ contains a point $\mu^{(0)}=\left(\mu_{1}^{(0)}, \ldots, \mu_{N-1}^{(0)}\right)$ at which $\operatorname{Re} e_{m}=\operatorname{Re} e_{m^{\prime}}$ for all $m, m^{\prime} ; \mathscr{U}$ should even contain a neighborhood of size $\geqq e^{-\tau / 2}$ about $\mu^{(0)}$. It will be understood in the following that any statement shall be restricted to $\mathscr{U}$.

In order to avoid inessential details in the construction, let us consider the representative case where $N=3$ and we are searching for a curve $\mu(t) \in \mathbb{R}^{2}$ on which $a_{1}=a_{2}=0$. Here $t \geqq 0$ and we wish further that $a_{3}(\mu(0))=0$ and $a_{3}(\mu(t))>0$ if $t>0$. Thus $\mu(0)$ is the point of maximal coexistence.

We first construct $\mu(0)$ as a limit of approximation coexistence points $\mu^{(0)}, \mu^{(1)}, \ldots$. The strategy is to consider neighborhoods

$$
\mathcal{N}^{(j)}=\left\{\mu \in \mathbb{R}^{2}:\left|\mu-\mu^{(j)}\right| \leqq\left(8 d_{j+1}\right)^{-1}\right\}
$$

$\left(d_{j}\right.$ is defined below and $|\cdot|$ denotes the maximum norm $\left.|\cdot|_{\infty}\right)$ and to construct $\mu^{(j+1)} \in \mathscr{N}^{(j)}$ in such a way that $\mathscr{N}^{(j+1)} \subset \mathscr{N}^{(j)}$. We introduce distance scales

$$
d_{0}=1, \quad d_{1}=e^{\tau d_{0} / 2}=e^{\tau / 2}, \ldots, d_{j+1}=e^{\tau d_{j} / 2},
$$

and contour functionals

$$
K^{(j)}\left(Y^{m}\right)= \begin{cases}K\left(Y^{m}\right) & \text { if } \operatorname{diam} Y^{m}<d_{j} \\ 0 & \text { otherwise }\end{cases}
$$


(Recall from Sect. 2 that $K$ is $\rho$ times a ratio of partition functions.) Our main assumption (to be proven inductively) is that

$$
a_{m}(\mu) d_{j} \leqq 1 \text { in } \mathscr{N}^{(j-1)}
$$

for all $m=1,2,3$. Then we have good control over the activities $K^{(j)}\left(Y^{m}\right)$, by Corollary 3.4. In particular, all $Y^{m}$ with diam $Y^{m} \leqq d_{j}$ are stable, $K^{(j)}\left(Y^{m}\right)$ is $C^{1}$ and

$$
\left|K^{(j)}\left(Y^{m}\right)\right|,\left|\frac{\partial}{\partial \mu_{i}} K^{(j)}\left(Y^{m}\right)\right| \leqq e^{-(1-\delta) \tau\left|Y^{m}\right|} .
$$

Here $\delta=1 / 10$ is fixed. These bounds imply that the free energies $s_{m}^{(j)}$ of the contour functionals $K^{(j)}\left(Y^{m}\right)$ are $C^{1}$ functions of $\mu$ in $\mathscr{N}^{(j-1)}$, satisfying bounds

$$
\begin{aligned}
&\left|s_{m}^{(j)}\right|,\left|\frac{\partial}{\partial \mu_{i}} s_{m}^{(j)}\right| \leqq e^{-(1-2 \delta) \tau} \\
&\left|S_{m}^{(j)}-s_{m}^{(j-1)}\right| \leqq e^{-(1-2 \delta) \tau d_{j}-1} \\
&\left|s_{m}^{(j)}-s_{m}\right| \leqq e^{-(1-2 \delta) \tau d_{j}}
\end{aligned}
$$

Finally, we introduce

$$
h_{m}^{(j)}=\operatorname{Re}\left(s_{m}^{(j)}+e_{m}\right) .
$$

Let $\mu^{(0)}$ be the point where $\operatorname{Re} e_{1}=\operatorname{Re} e_{2}=\operatorname{Re} e_{3}$, or equivalently $h_{1}^{(0)}=h_{2}^{(0)}=h_{3}^{(0)}$. It is unique, by the degeneracy-removing condition. Let $\mathscr{N}^{(0)}$ be the neighborhood of $\mu^{(0)}$ of radius $\left(8 d_{1}\right)^{-1}$. In $\mathscr{N}^{(0)}$ we have by $(6.2),(3.9)$ that

$$
\begin{aligned}
a_{m} & \leqq \max _{m^{\prime}}\left\{\left|h_{m}^{(0)}-h_{m^{\prime}}^{(0)}\right|+\left|s_{m}\right|+\left|s_{m^{\prime}}\right|\right\} \\
& \leqq \frac{1}{2 d_{1}}+O\left(e^{-\tau}\right) \leqq \frac{1}{d_{1}}
\end{aligned}
$$

Hence $a_{m} d_{1} \leqq 1$ and we can consider $h_{m}^{(1)}$. Note that $\operatorname{Re} s_{m}^{(1)}=h_{m}^{(1)}-\operatorname{Re} e_{m}$ is controlled by (6.5a), so nondegeneracy holds for $h_{m}^{(1)}$, with a constant slightly larger than $M$. Note that by $(6.5 \mathrm{~b})\left|h_{m}^{(1)}\left(\mu^{(0)}\right)-h_{m}^{(0)}\left(\mu^{(0)}\right)\right|=O\left(e^{-(1-2 \delta) \tau}\right) \ll \operatorname{Radius} \mathscr{N}^{(0)}$. Hence there is a point $\mu^{(1)}$ in $\mathscr{N}^{(0)}$, where $h_{1}^{(1)}=h_{2}^{(1)}=h_{3}^{(1)}$. Even the neighborhood $\mathscr{N}^{(1)}$ of $\mu^{(1)}$ of radius $\left(8 d_{2}\right)^{-1}$ is contained in $\mathscr{N}^{(0)}$.

It is clear now how to proceed to construct the $\mu^{(j)}$ and the corresponding neighborhoods $\mathscr{N}^{(j)}$ of $\mu^{(j)}$ of radius $\left(8 d_{j+1}\right)^{-1}$. It is important to check the condition (6.4) that $a_{m} d_{j+1} \leqq 1$ in $\mathscr{N}^{(j)}$. In $\mathscr{N}^{(j-1)}$ the free energies $h_{m}^{(j)}$ were considered by truncation at contour diameter $d_{j}$, with $a_{m} d_{j} \leqq 1$. Therefore, by (6.5),

$$
\begin{aligned}
a_{m} & \leqq \max _{m^{\prime}}\left\{\left|h_{m}^{(j)}-h_{m^{\prime}}^{(j)}\right|+\left|h_{m}-h_{m}^{(j)}\right|+\left|h_{m^{\prime}}-h_{m^{\prime}}^{(j)}\right|\right\} \\
& \leqq \frac{3}{4 d_{j+1}}+2 e^{-\tau(1-2 \delta) d_{j}} \leqq \frac{1}{d_{j+1}} .
\end{aligned}
$$

The first term was estimated from the uniform bound $\left|\partial h_{m}^{(j)} / \partial \mu_{i}\right| \leqq \frac{3}{2}$ and the fact that $h_{m}^{(j)}=h_{m^{\prime}}^{(j)}$ at the center of $\mathscr{N}^{(j)}$. Finally,

$$
\left|h_{m}^{(j+1)}\left(\mu^{(j)}\right)-h_{m}^{(j)}\left(\mu^{(j)}\right)\right| \leqq e^{-(1-2 \delta) d_{j}} \ll \operatorname{Radius} \mathscr{N}^{(j)},
$$


so it is possible to find the point $\mu^{(j+1)}$ at which $h_{1}^{(j+1)}=h_{2}^{(j+1)}=h_{3}^{(j+1)}$ and such that $\mathscr{N}^{(j+1)} \subset \mathscr{N}^{(j)}$.

It is clear that the sequence $\mu^{(j)}$ converges very rapidly and that at the limit [defined to be $\mu(0)]$ we have $a_{1}=a_{2}=a_{3}=0$.

We consider now the curve $\mu(t)$. At the beginning we can solve for a curve $\mu^{(0)}(t)$ starting at $\mu^{(0)}$ and such that

$$
h_{1}^{(0)}=h_{2}^{(0)}=h_{3}^{(0)}-t .
$$

This is a $C^{1}$ curve, by the implicit function theorem. We restrict attention to the strip $\mathscr{S}^{(0)}$, neighborhood of the curve of radius $\left(8 l_{1}\right)^{-1}$, with $l_{1} \geqq d_{1}$ to be chosen in a moment. In $\mathscr{S}^{(0)}$ we can estimate $a_{1}$ and $a_{2}$ by $1 / d_{1}$ as in (6.7), but not $a_{3}$. To be more precise, we first note that for $\mu=\mu^{(0)}(t) \notin \mathcal{N}^{(0)}, h_{3}^{(0)}>h_{2}^{(0)}+1 / 8 M d_{1}$ by the degeneracy-removing condition. Therefore, by (6.2) and for a suitable choice of $l_{1}$ (e.g. $l_{1}=16 M d_{1}$ ), we have then $h_{3}^{(0)}>h_{2}^{(0)}+O\left(1 / d_{1}\right)$ in $\mathscr{S}^{(0)} \backslash \mathscr{N}^{(0)}$. Since $\operatorname{Re} h_{m}-h_{m}^{(0)}=O\left(e^{-\tau}\right) \ll 1 / d_{1}$ in the whole region $\mathscr{U}$, we conclude that

$$
\operatorname{Re} h_{3}>\operatorname{Re} h_{2}+O\left(1 / d_{1}\right) \text { in } \mathscr{S}^{(0)} \backslash \mathscr{N}^{(0)} \text {. }
$$

So state 3 is well out of contention for the smallest $h$ in $\mathscr{S}^{(0)} \backslash \mathscr{N}^{(0)}$. Thus we restrict $m^{\prime}$ in (6.7) to $\{1,2\}$.

Since we have no bound on $a_{3}$ in $\mathscr{S}^{(0)}$, we may not consider $h_{3}^{(1)}$; it does not provide a parametrization for $\mu^{(1)}(t)$. Instead we solve for the curve $\mu^{(1)}(t)$ such that

$$
h_{1}^{(1)}=h_{2}^{(1)}=h_{3}^{(0)}-h_{3}^{(0)}\left(\mu^{(1)}\right)+h_{3}^{(1)}\left(\mu^{(1)}\right)-t .
$$

When $t=0$, this equation is satisfied by $\mu^{(1)}(0)=\mu^{(1)}$. Note that nondegeneracy holds for $\left\{h_{1}^{(1)}, h_{2}^{(1)}, h_{3}^{(0)}\right\}$, so we can use the implicit function theorem to solve this equation, obtaining a differentiable $\mu^{(1)}(t)$. We only have to check that $\mu^{(0)}(t)$ solves (6.10) sufficiently accurately so that $\mu^{(1)}(t)$ can be found in $\mathscr{S}^{(0)}$. The errors are $O\left(e^{-\tau(1-2 \delta)}\right) \ll \operatorname{Radius} \mathscr{S}^{(0)}$, so we have $\mu^{(1)}(t)$.

The general step works as in the construction of $\mu^{(j+1)}$, only we solve Eq. (6.10) with 1 replaced by $j+1$. This can be written as

$$
h_{1}^{(j+1)}=h_{2}^{(j+1)}=h_{3}^{(0)}+\operatorname{Re} s_{3}^{(j+1)}\left(\mu^{(j+1)}\right)-t,
$$

and we look for the solution curve $\mu^{(j+1)}(t)$. We first check that

$$
\operatorname{Re} h_{3}>\operatorname{Re} h_{2}+O\left(1 / d_{j+1}\right) \text { in } \mathscr{S}^{(j)} \backslash \mathscr{N}^{(j)},
$$

where the strip $\mathscr{S}^{(j)}$ is a neighborhood of the curve $\mu^{(j)}(t)$ of radius $\left(8 l_{j+1}\right)^{-1}$, with $l_{j+1}=16 M d_{j+1}$. Since (6.12) has been checked in the previous step for $j-1$ instead of $j$, and $\mathscr{S}^{(j)} \subset \mathscr{S}^{(j-1)}$, we only have to consider $\mu \in \mathscr{S}^{(j)} \cap \mathscr{N}^{(j-1)} \backslash \mathscr{N}^{(j)}$. As above we first consider $\mu=\mu^{(j)}(t) \in \mathscr{N}^{(j-1)} \backslash \mathscr{N}^{(j)}$. We use the degeneracy-removing condition for $\left(h_{1}^{(j)}, h_{2}^{(j)}, h_{3}^{(0)}\right)$ and the fact that

$$
\left|s_{3}^{(j)}(\mu)-s_{3}^{(j)}\left(\mu^{(j)}\right)\right| \leqq\left|\mu-\mu^{(j)}\right| O\left(e^{-\tau(1-2 \delta)}\right)
$$

if $\mu \in \mathscr{N}^{(i-1)}$. As a consequence we have for $\mu=\mu^{(j)}(t) \in \mathscr{N}^{(j-1)} \backslash \mathscr{N}^{(j)}$ that

$$
h_{2}^{(j)}\left(\mu^{(j)}(t)\right)<h_{3}^{(j)}\left(\mu^{(j)}(t)\right)-\frac{1}{16 M d_{j+1}} .
$$


Using the uniform bound $\left|\partial h_{m}^{(j)} / \partial \mu_{i}\right| \leqq 3 / 2$ and the bound (6.5) for $\operatorname{Re} h_{m}-h_{m}^{(j)}$ we obtain (6.12) in the region $\mathscr{S}^{(j)} \cap \mathscr{N}^{(j-1)} \backslash \mathscr{N}^{(j)}$.

Due to (6.12), $a_{1}$ and $a_{2}$ can be estimated by restricting $m^{\prime}$ in (6.8) to $\{1,2\}$ [in $\mathscr{N}^{(j)}$, the necessary bound on $a_{1}, a_{2}$ has already been verified in the construction of $\mu(0)]$. The curve $\mu^{(j)}(t)$ solves $(6.11)$ up to errors $O\left(e^{-(1-2 \delta) \tau d_{j}}\right)$ because $\left|h_{1}^{(j+1)}-h_{1}^{(j)}\right|, \quad\left|h_{2}^{(j+1)}-h_{2}^{(j)}\right|, \quad\left|s_{3}^{(j+1)}\left(\mu^{(j)}\right)-s_{3}^{(j)}\left(\mu^{(j)}\right)\right|, \quad$ and $\left|\mu^{(j+1)}-\mu^{(j)}\right|$ are all $O\left(e^{-(1-2 \delta) \tau d_{j}}\right)$. Thus we can continue inductively, obtaining a sequence of curves $\mu^{(j)}(t)$, which are $C^{1}$ uniformly in $j$. Equation (6.11) tends to a limit, so the curves converge for each $t$ to a limit $\mu(t)$, which moreover is differentiable. (To get a $C^{1}$ limit curve, we naturally have to assume uniform continuity of derivatives throughout.)

On the limit curve, the statement that state 3 is out of contention in each $\mathscr{S}^{(j)} \backslash \mathscr{N}^{(j)}$ translates into a statement that $a_{3}>0$ for $t>0$. Of course $a_{1}=a_{2}=0$ on the curve $\mu(t)$. Thus we have produced the desired portion of the phase diagram. It should be clear now how to proceed to obtain any portion of the diagram for the $N$-state problem.

Acknowledgements. The authors would like to thank G. Velo and A. Wightman for their hospitality at the Centro Ettore Majorana, Erice, where much of this work was carried out. C.B. would like to thank R. Waxler for many illuminating discussions.

\section{References}

[BG] Balaban, T., Gawedzki, K.: A low-temperature expansion for the pseudoscalar Yukawa model of quantum fields in two space-time dimensions. Ann. Inst. Henri Poincaré 36, 271-400 (1982)

[BW] Borgs, C., Waxler, R.: First-order phase transitions in unbounded spin systems. Preprint

[BKL] Bricmont, J., Kuroda, K., Lebowitz, J. L.: First-order phase tansitions in lattice and continuous systems: Extension of Pirogov-Sinai theory. Commun. Math. Phys. 101, 501-538 (1985)

[DZ] Dobrushin, R. L., Zahradnik, M.: In: Mathematical problems of statistical physics and dynamics. Dobrushin, R. L. (ed.). Dortrecht: Reidel 1986

[GKK] Gawedzki, K., Kotecký, R., Kupiainen, A.: Coarse-graining approach to first-order phase transitions. J. Stat. Phys. 47, 701-724 (1987)

[I] Imbrie, J. Z.: Phase diagrams and cluster expansions for low temperature $\mathscr{P}(\phi)_{2}$ models. I. Commun. Math. Phys. 82, 261-304 (1981). II. Commun. Math. Phys. 82, 305-343 (1981)

[JLW] Jaffe, A., Lesniewski, A., Weitsman, J.: Index of a family of Dirac operators on loop space. Commun. Math. Phys. 112, 75 (1987), The loop space $S^{1} \rightarrow \mathbb{R}$ and supersymmetric quantum fields. Ann. Phys. 183, 337-351 (1988)

[PS] Pirogov, S., Sinai, Ya.: Phase diagrams of classical lattice systems. Theor. Math. Phys. 25, 1185-1192 (1975) and 26, 39-49 (1976)

[W] Weitsman, J.: A supersymmetric field theory in infinite volume. Harvard Ph.D. thesis. (1988)

[Z1] Zahradnik, M.: An alternative version of Pirogov-Sinai theory. Commun. Math. Phys. 93, 559-581 (1984)

[Z2] Zahradnik, M.: In: Statistical mechanics and field theory: Mathematical Aspects (Groningen 1985). Dorlas, T. C., Hugenholtz, N. M., Winnick, M. (eds.). Berlin, Heidelberg, New York: Springer 1986

[Z3] Zahradnik, M.: Analyticity of low-temperature phase diagrams of lattice spin models. J. Stat. Phys. 47, 725-755 (1987)

Communicated by A. Jaffe

Received October 17, 1988 